\title{
La Cicogna bianca (Ciconia ciconia) in Piemonte e in Provincia di Torino dal 1996 al 2014
}

\author{
Paolo Marotto $^{1 *}$, Luciano Ruggieri ${ }^{2}$, Gabriella Vaschetti ${ }^{3}$
}

\begin{abstract}
Riassunto - Viene presentata un'analisi della popolazione di Cicogna bianca (Ciconia ciconia) in Piemonte nord occidentale e, specificatamente, in provincia di Torino negli ultimi 20 anni. Il primo tentativo di nidificazione risale al 1980, ma è solo a partire dal 1996 che si è registrata una colonizzazione del territorio torinese con la presenza di un massimo di 8 coppie (2014). Parallelamente, anche il numero di individui svernanti nella stessa area, particolarmente in prossimità di discariche di rifiuti urbani, è aumentato. Viene indicata una correlazione tra l'aumento del numero di individui non migranti e il numero di coppie nidificanti nella stessa area.
\end{abstract}

Parole chiave: Cicogna bianca, status, Piemonte, provincia di Torino, N-O Italia

\begin{abstract}
The white stork (Ciconia ciconia) in Piedmont and in the province of Turin, from 1996 to 2014.

We analysed the white stork (Ciconia ciconia) population in northwest Piedmont and, specifically, in the province of Turin over the last 20 years. The first attempt at nesting dates back to 1980, but only since 1996 has there been settlement in the Turin area, with the presence of a maximum of 8 pairs. At the same time, the number of individuals wintering within the area, particularly in the vicinity of municipal waste landfills, increased. We show a correlation between the number of non-migrant white storks and the number of breeding pairs in the given area.
\end{abstract}

Key words: White Stork, status, Piedmont, Turin, N-W Italy.

\section{INTRODUZIONE}

La Cicogna bianca (Ciconia ciconia) è in Italia una specie nidificante rara con una distribuzione molto frammentata e ristretta principalmente alla Pianura Padana nord-occidentale (Brichetti \& Fracasso, 2003). La prima nidificazione certa in Italia è, infatti, del 1959 nella pro-

\footnotetext{
${ }^{1}$ Via Monterosa 1, 10099 San Mauro Torinesese (TO), Italia.

${ }^{2}$ Via Buniva 2, 10124 Torino, Italia.

E-mail: ruggiel@libero.it

${ }^{3}$ Centro Cicogne Racconigi (CN), Italia.

E-mail: gvaschetti@libero.it

* Corrisponding author: paolomarotto2@gmail.com

(C) 2017 Paolo Marotto, Luciano Ruggieri, Gabriella Vaschetti
}

Received: 8 December 2015

Accepted for publication: 23 October 2017 vincia di Vercelli (Toschi, 1960), dove negli anni '90 si contavano 12 coppie (Vanzi, Ricci \& Bottelli, 1994). La popolazione italiana, stimata in 88 individui maturi $(\mathrm{Gu}-$ stin \& Tallone, 2005) è attualmente in forte incremento (Brichetti \& Fracasso, 2003; BirdLife International, 2004) in seguito a colonizzazioni spontanee e azioni di protezione specifiche. Considerata a "minore preoccupazione" nella Lista Rossa 2011 degli Uccelli Nidificanti in Italia è ora valutata in 150-200 coppie nel Rapporto sull'applicazione della Direttiva 147/2009/CE in Italia (2008-2012).

La comprensione dei fattori che influenzano la dinamica di popolazione e la distribuzione spaziale di specie rare o minacciate presuppone una conoscenza approfondita dei fattori ecologici che ne regolano la biologia riproduttiva e i movimenti migratori. Tali conoscenze sono spesso utilizzate per definire al meglio la gestione ai fini della conservazione o per incrementare localmente le popolazioni (Verner et al., 1986).

La Cicogna bianca è una specie migratrice che ogni anno compie spostamenti tra le aree di riproduzione in Europa e quelle di svernamento, poste prevalentemente nell'Africa sud sahariana, transitando lungo due rotte principali: a est attraverso il Bosforo e a ovest sullo stretto di Gibilterra. Assente quindi storicamente come svernante in Italia, la specie ha fatto registrare in anni recenti una tendenza a permanere nelle aree di nidificazione, fenomeno sicuramente incrementato dalla presenza di individui proveniente da centri di reintroduzione tendenzialmente meno propensi ad abbandonare le aree di rilascio e reintroduzione.

Questo lavoro prende in esame lo status della Cicogna bianca in Piemonte ed in particolare in provincia di Torino come specie nidificante e recente svernante, con l'obiettivo di proporre più razionali misure di conservazione $\mathrm{e}$ tutela.

\section{MATERIALI E METODI}

Sono stati raccolti dati sulla presenza della specie tramite indagine bibliografica e osservazioni dirette. La ricerca sul campo è stata svolta dagli autori a partire 2005 e realizzata anche grazie ai dati pervenuti alla lista e al sito web di Torino Birdwatching. Le aree sono state selezionate in base a quelle ritenute più idonee all'insediamento della specie in Provincia di Torino. La spiccata filopatria della specie ha permesso di concentrare le ricerche in aree 
viciniori a quelle con coppie nidificanti. Dati di terzi sono stati verificati direttamente con visite in situ. Alcuni dei nidi artificiali installati in Provincia sono stati posti in sede tramite accordi con l'Ente Parco Naturale La Mandria. I dati bibliografici sono quelli dell'archivio del Gruppo Piemontese Studi Ornitologici (GPSO) e dei Re.P.Or.T. (Resoconto Provinciale Ornitologico Torinese), che raccolgono anche i dati di Torino Birdwatching.

\section{RISULTATI}

\section{Situazione in Piemonte}

Le prime nidificazioni in tempi recenti documentate in Regione, ritenute anche le prime registrate in assoluto per la penisola italiana, sono riportate da G. Boano e risalgono al 1959 (Riv. Piem. Storia Nat., 1981). Sempre da Boano si apprende che già il Toschi citava la presenza di coppie nidificanti nel Biellese 4 o 5 anni prima (Toschi, 1960) e riportava la nidificazione di tre coppie ai margini di una riserva di caccia a Rovasenda (VC). In seguito le segnalazioni di riproduzioni o tentativi di costruzione del nido si limitarono sempre alla presenza di singole coppie. Nella maggior parte dei casi le deposizioni non andarono a buon fine a causa dell'abbattimento dei soggetti o al disturbo antropico.

L'atlante degli uccelli nidificanti in Piemonte (19801984) riportava solo 4 nidificazioni e, in effetti, fino verso la fine degli anni ottanta non vi furono cambiamenti sostanziali riguardo lo status della Cicogna bianca in Italia, come riportato da Tallone et al. nel 1993 e dallo stesso Boano, che nel 1992 citava scarsi casi di insediamento, perchè pochi superavano lo stadio iniziale di costruzione del nido (Boano, 1992).

Un deciso incremento si ebbe a partire dal 1985 quando in Piemonte venne avviato un importante progetto di reintroduzione in natura, sotto la supervisione scientifica dell'ornitologo Fabio Perco, per merito di Bruno Vaschetti in collaborazione con la LIPU presso il Centro Cicogne di Racconigi $(\mathrm{CN})$.
Nel 1988 a seguito di diversi tentativi di nidificazione falliti negli anni precedenti nella provincia di Novara (presso lo zoo-safari di Pombia ed a Gionzana nel 1996), una coppia si riprodusse con successo nel vercellese a Balocco. Nel 1989 almeno 4 coppie nidificarono anche nel novarese a Vicolungo, Casalgiate, Agrate e Conturbia (in GPSO). Altre si limitarono alla costruzione del nido.

Nel 1990 si registrarono 8 coppie nidificanti sul territorio regionale: 2 in provincia di Cuneo, 3 in quella di Vercelli e 3 di Novara.

Negli anni successivi venne osservato un costante incremento del numero di nidificazioni all'interno del Centro Cicogne di Racconigi. Nel 1991 venne registrata la prima riproduzione di una coppia mista (individui in semilibertà e selvatici) e nel 1992 quella composta esclusivamente da soggetti di origine selvatica. Da notare che già nel 1992 le coppie nidificanti in tutto il Piemonte involarono ben 30 giovani che rappresentavano allora oltre la metà dei giovani allevati in Italia (Tallone et al., 1993).

Il resoconto ornitologico per il Piemonte e Valle d'Aosta del 1993 riportava per il Piemonte una coppia nel cuneese (oltre a quelle presenti nel Centro Lipu di Racconigi), 5 nel vercellese e 6 nel novarese (Alessandria et al., 1994).

Gariboldi nel 1995 affermava che la situazione della Cicogna bianca in Italia, pur se non ancora stabilizzata, era decisamente mutata in maniera rapida e che la specie poteva ritenersi nidificante regolare, almeno per l'area compresa nella Pianura Padana centro occidentale (Lui, 2004).

Tra le province di Cuneo e Torino nel 1996 nidificarono 20 coppie di cui 7 formate da individui reintrodotti, 8 miste e 5 selvatiche; 19 arrivarono alla deposizione e 10 involarono in totale 19 pulli (Vaschetti et al., 1997).

Nel 1997 in Piemonte erano presenti 31 coppie, 21 nelle province di Torino e Cuneo con coppie formate in 10 casi da individui reintrodotti, in 6 casi da coppie miste e in 5 di origine selvatica. Venne accertata la deposizione in 17 nidi e la nascita di 44 pulli in totale (Vaschetti et al., 1999).

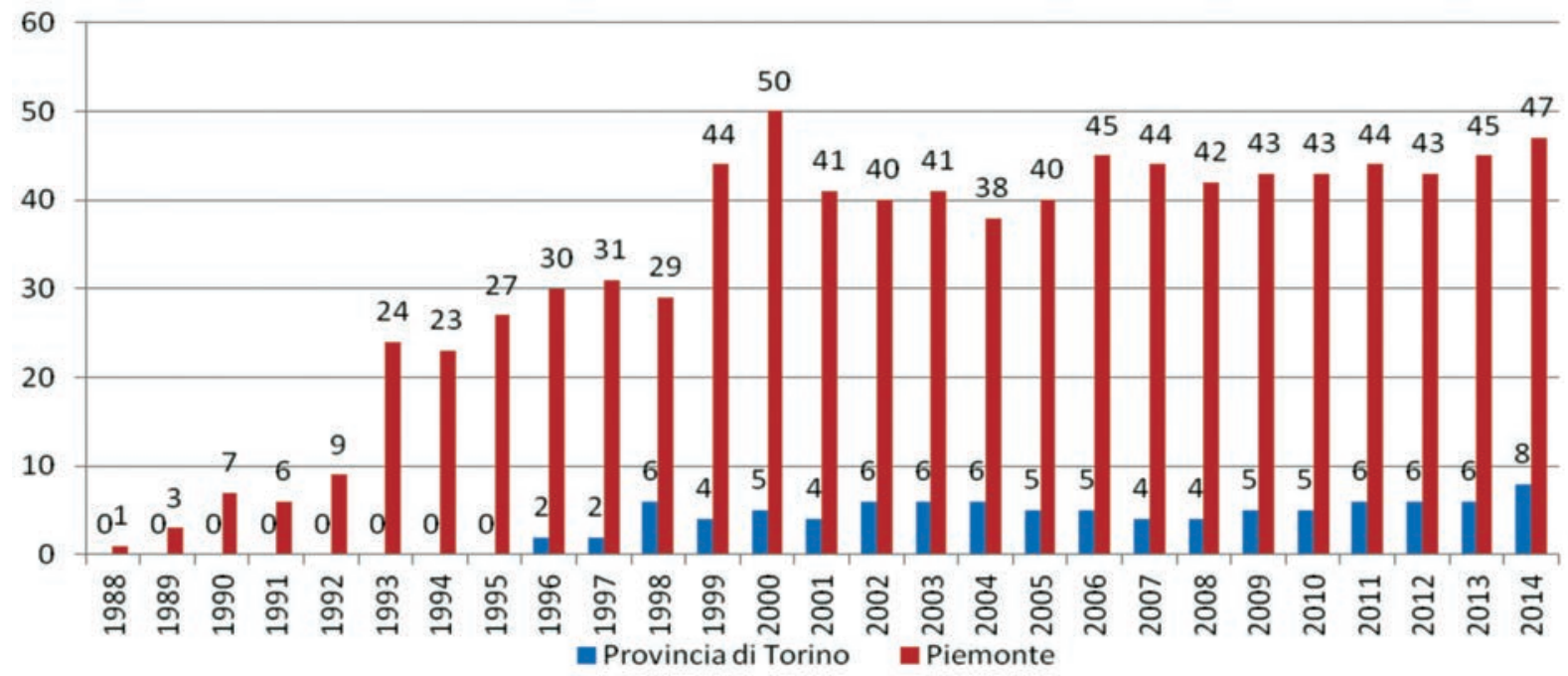

Fig. 1 - Numero di coppie nidificanti in Piemonte (rosso) e in provincia di Torino (azzurro). / Number of breeding pairs in Piedmont (red columns) and in the province of Turin (in blue). 


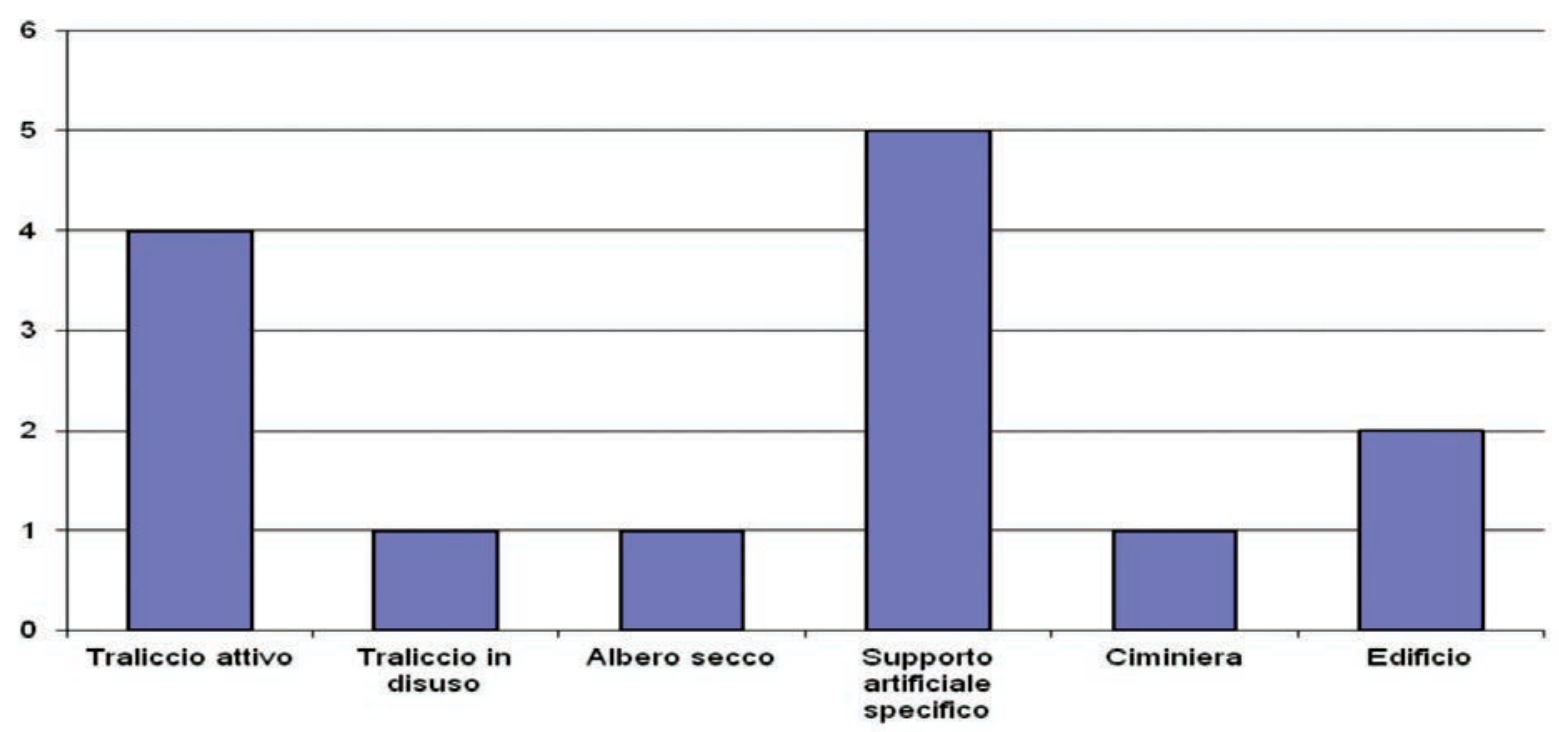

Fig. 2 - Tipologia di struttura utilizzata per la nidificazione. / Type of structures on which the nests are built: used trellises, disused trellises.

Nel 1998 per le sole due province occidentali piemontesi erano 29 quelle certe composte in 13 casi da individui reintrodotti, in 6 di origine mista e in 10 selvatica; venne accertata la deposizione in 20 casi e la nascita di 50 pulli (Vaschetti et al., 1999)

Nell'aggiornamento della distribuzione dei nidificanti in Piemonte (1994-1997) il numero totale delle coppie conosciute oscillava tra 23 e 41 (Aimassi \& Reteuna, 2007).

Nel 2000 ne vennero conteggiate 50: 34 in provincia di Cuneo (Caula \& Beraudo, 2014), 6 di Biella, 3 di Novara, 3 di Torino e 4 di Vercelli (F. Carpegna, Centro Cicogne Racconigi ed altri oss.). Nel 2001 la Cicogna bianca era presente con 41 coppie: 26 in provincia di Cuneo, 4 a Torino, 6 a Biella, 3 a Novara e 2 a Vercelli (in GPSO).

In una indagine del 2002 venne registrato un leggero calo del numero di nidificanti: le coppie in Piemonte risultavano 40 e rappresentavano il 39 per cento dell'intera popolazione nazionale (Vaschetti \& Boano, 2005).

In seguito la popolazione si consolidò intorno alle 3845 coppie registrate a partire dal 2003/2004 (Alessandria et al., 2003; 2004). Nel 2004 delle 38 coppie presenti sul territorio piemontese, 21 nidificarono, portando all'involo 49 giovani (Gustin \& Tallone, 2005).

In provincia di Cuneo la popolazione nidificante si è ormai stabilizzata, annoverando una trentina di coppie, presenti soprattutto a Racconigi, sia presso il Centro Cicogne sia presso la città, dove le cicogne hanno colonizzato le sommità di chiese ed edifici storici. Singole coppie sono presenti nei comuni limitrofi: Caramagna Piemonte, Cavallermaggiore, Savigliano (fraz. Levaldigi) e Bra.

Se da un lato è stata favorevole la colonizzazione spontanea di siti al di fuori delle piattaforme dell'oasi, dall'altro ciò ha comportato una netta diminuzione dell'attività di inanellamento con anelli colorati forniti dall'INFS (ora ISPRA), per l'inaccessibilità ai nidi, diminuendo così i casi di lettura durante gli spostamenti migratori.

\section{Nidificazione in provincia di Torino}

Per la provincia di Torino dopo il 1980 si hanno segnalazioni di Cicogna bianca in periodo riproduttivo nella Vauda Canavesana (3 individui a Lombardore il 18 maggio 1981). Il primo tentativo di nidificazione per il torinese risale al 1980 a San Giovanni di Carmagnola e fallì presumibilmente a causa dell'uccisione di uno dei due individui ritrovato morto a Scarnafigi a circa 20 $\mathrm{km}$ dal punto in cui era stato costruito il nido (Boano, 1981). Nel 1996 fu registrata la prima nidificazione di soggetti in semilibertà all'interno del Parco Ornitologico "Martinat" di San Pietro in Val di Lemina (Pinerolo); nello stesso anno si assistette all'insediamento di una coppia, apparentemente selvatica, in un'area limitrofa (Tab. 1).

Dal 2004 al 2014 sono stati raccolti i dati relativi a 16 nidi in provincia di Torino. Il numero medio di giovani involati è risultato di 1.125 , nel $31 \%$ dei casi è stato allevato un solo pullo, in uno solo 4. La percentuale di nidificazioni fallite per varie cause, soprattutto metereologiche, è complessivamente del $31.25 \%$.

Nel 2006 la lettura di un anello colorato posto alla zampa di uno dei due soggetti nidificanti a Gassino permise di risalire alla sua origine francese in seguito anche il giovane allevato dalla coppia venne inanellato nel nido. Le date di insediamento piuttosto precoci rilevate a Gassino e Volpiano, con individui che stazionano sul nido già dalla metà del mese di febbraio, fanno supporre che gli individui svernino in loco; comportamento già osservato nei soggetti stanziali dell'Europa continentale, con nidificazioni alla fine dell'inverno, alcune volte già a partire dalla metà di dicembre (Belardi et al., 2004).

La tipologia di struttura utilizzata per la costruzione dei nidi in Provincia di Torino, come indicato per il Piemonte da Vaschetti et al. 1997, è principalmente artificiale. Con la collaborazione di EBN Italia, della LIPU di Torino e dell'Associazione Naturalistica Le Gru 
Tab. 1 - Numero di coppie di Cicogna bianca nidificanti in Provincia di Torino tra il 1996 al 2014. / Number of breeding pairs in the province of Turin between 1996 and 2014.

\begin{tabular}{|c|c|c|c|c|c|c|c|c|}
\hline Anno & Pinerolese & Villafranca P.te & La Motta & Gassino & Volpiano & Lombardore & Bosconero & Totale \\
\hline 1996 & 2 & & & & & & & 2 \\
\hline 1997 & 2 & & & & & & & 2 \\
\hline 1998 & 5 & 1 & & & & & & 6 \\
\hline 1999 & 4 & & & & & & & 4 \\
\hline 2000 & 5 & & & & & & & 5 \\
\hline 2001 & 4 & & & & & & & 4 \\
\hline 2002 & 6 & & & & & & & 6 \\
\hline 2003 & 6 & & & & & & & 6 \\
\hline 2004 & 5 & & 1 & & & & & 6 \\
\hline 2005 & 3 & & & & & & & 5 \\
\hline 2006 & 4 & & & 1 & & & & 5 \\
\hline 2007 & 3 & & & 1 & & & & 4 \\
\hline 2008 & 3 & & & 1 & & & & 4 \\
\hline 2009 & 3 & & & 1 & 1 & & & 5 \\
\hline 2010 & 3 & & & 1 & 1 & & & 5 \\
\hline 2011 & 3 & & & 1 & 1 & 1 & & 6 \\
\hline 2012 & 3 & & & 1 & 1 & 1 & & 6 \\
\hline 2013 & 2 & & & 1 & 1 & 1 & 1 & 6 \\
\hline 2014 & 3 & & & 1 & 2 & 1 & 1 & 8 \\
\hline
\end{tabular}

quattro nidi artificiali vennero collocati dall' Ente Parco all'interno del Parco Naturale Regionale della Mandria e nell'Oasi del L ago Borgarino. Attualmente nessun tentativo di nidificazione è stato riportato per queste strutture.

Alti alberi morti vengono sfruttati anche se all'interno di aree fortemente antropizzate, come è stato riscontrato per la coppia di Gassino, all'inizio del suo insediamento.

\section{Lo svernamento in Piemonte}

La stima del numero di siti di svernamento in Italia ha visto un rapido incremento ed è passato dai 4 siti censiti nel 1991-95 ai 143 del 2006-10, facendo registrare un trend decennale di $+9.7 \%$ annuo e del $+27 \%$ sul lungo periodo (Zenatello et al., 2014). In Piemonte dopo le osservazioni tardive registrate nel 1981, la presenza invernale della specie venne segnalata per la prima volta nell'alessandrino il 28 dicembre 1985 a Bozzole (AL), (in GPSO). Il primo caso documentato di svernamento effettivo di individui si riferisce a 2 soggetti dal 21 ottobre 1997 al 22 febbraio 1998 presenti tra Cerano e Tornaco (NO) (in GPSO).

\section{Lo svernamento in provincia di Torino}

Tra i primi casi di presenza invernale della specie in Provincia, si segnalano un individuo a Perosa Canavese il
1 febbraio 1994 (A. Pedrazzo in GPSO) e uno nel Parco Regionale La Mandria il 4 dicembre 1994 (M. Doimo in GPSO).

Lo svernamento regolare è stato registrato dal 2000 in prossimità delle aree frequentate durante la riproduzione o poste nelle immediate vicinanze di disponibilità trofiche come le discariche di rifiuti urbani a Pinerolo e Torino (in GPSO). Presenze numeriche in incremento negli anni a partire dall'autunno e protratte per tutto il periodo invernale. In Fig. 3 è presentato il numero di soggetti svernanti presso le discriche di Basse di Stura e Cassagna (Torino) nel periodo $2004-2015$.

Con la chiusura, nel dicembre del 2009, dell'impianto di smaltimento di Basse di Stura, nel periodo invernale si è osservato l'immediato abbandono dell'area da parte delle cicogne e il successivo sfruttamento di un altro sito nei pressi di un impianto di smaltimento a Pianezza. La mancata lettura di anelli identificativi non ha permesso di verificare l'effettiva appartenenza dei singoli individui alla popolazione locale nidificante ma, la presenza regolare di soggetti nei pressi dei nidi di Volpiano e Gassino (Marotto, 2014) fa ipotizzare che almeno parte dei riproduttori sverni in loco. L'origine degli individui svernanti è stata determinata una sola volta grazie alla lettura di un anello colorato posto alla zampa di un soggetto inanellato a Fagagna (UD) il 25 settembre 2009 ed osservato presso la discarica di Basse di Stura l'11 novembre dello stesso anno. 


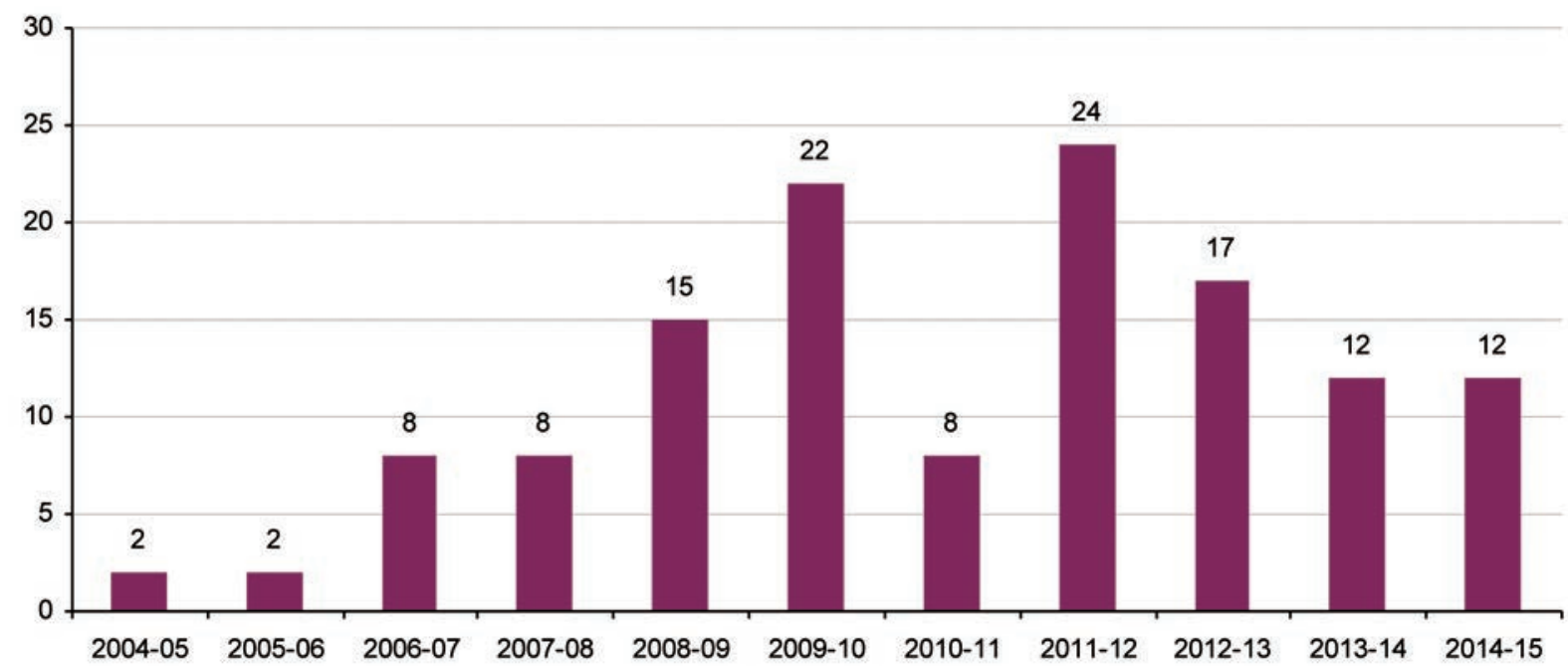

Fig. 3 - Numero di individui presenti d'inverno (dicembre - gennaio ) nell' area delle discariche di Basse di Stura e Cassagna. / Number of White storks present in winter (December and January) in the garbage dumps of Basse di Stura and Cassagna.

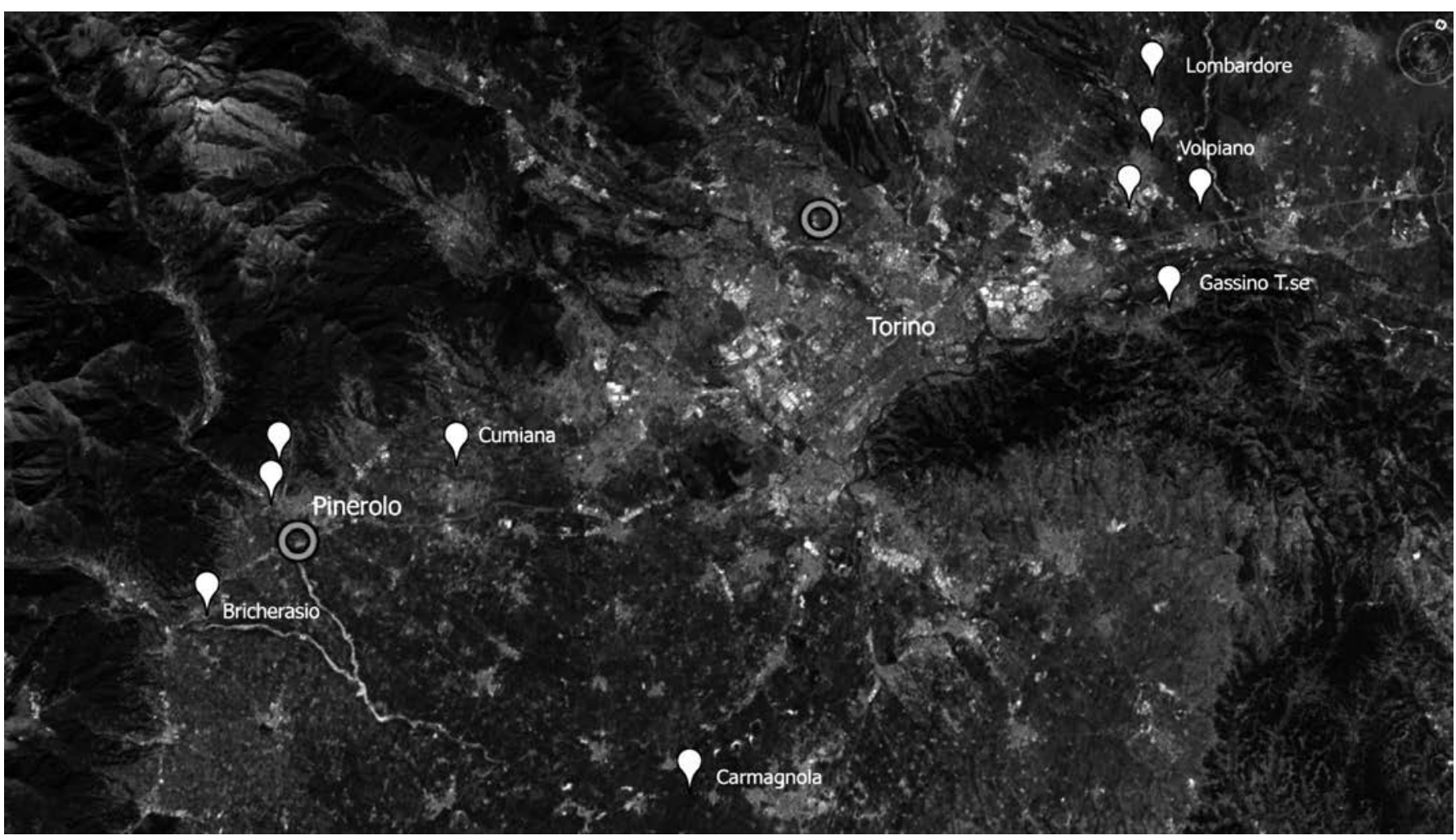

Fig. 4 - Localizzazione dei siti riproduttivi (bianco) e di svernamento (cerchio) in provincia di Torino. / Nesting (white) and wintering (circle) sites in the province of Turin.

\section{DISCUSSIONE}

La Cicogna bianca è un esempio di specie antropofila, la cui biologia è legata strettamente alle attività umane. Estinta in Italia a causa della persecuzione diretta, il suo recente incremento è dipendente dal maggior grado di tolleranza e di protezione da parte dell'uomo.

L'apertura di progetti specifici di salvaguardia, come quello della LIPU intrapreso a Racconigi, ha permesso alla specie di conquistare nuovi territori di nidificazione, data anche la sua spiccata filantropia.
Come già evidenziato da numerosi studi (ad es. Belardi, Canziani e Dimitolo), i fattori limitanti la diffusione della Cicogna bianca in Italia sono molteplici e vanno individuati in primis nella perdita legata all'inarrestabile espansione dei centri urbani e delle aree industriali che frammentano un territorio già degradato e che influiscono negativamente sulle disponibilità trofiche. La specie nidifica e frequenta aree per l'alimentazione prossime agli insediamenti umani; inoltre costruisce nidi molto visibili e localizzabili e può essere facilmente vittima di atti di 
bracconaggio, che soprattutto in passato, hanno condizionato il suo insediamento.

Attualmente la popolazione di Cicogna bianca nel torinese è distinta in due centri di irradiazione principali. Uno intorno a Pinerolo, a nord ovest del capoluogo, un altro a est, nel Basso Canavese, tra il fiume Po e il torrente Orco. Il primo è originato da una coppia semi domestica che nidificava presso il Parco Ornitologico Martinat, mentre la seconda da uno spontaneo insediamento a Gassino T.se di una coppia costituita da un soggetto di origine francese e da uno senza anelli.

La presenza di individui stanziali reintrodotti legati ai siti di rilascio, come per il Parco Ornitologico Martinat, chiuso poi nel 2009, e la vicinanza con i confini provinciali del Centro Cicogne di Racconigi, hanno spinto individui di origine selvatica a insediarsi sul territorio. La presenza di fonti trofiche e in particolare di appezzamenti coltivati a riso, anche se di limitata estensione, rappresenta un fattore particolarmente favorevole all'insediamento: le nidificazioni nel Canavese, di Gassino e Bosconero, sono avvenute in stretta vicinanza di risaie. A conferma che tale tipologia di habitat risulta idonea per la ricerca del cibo (rane e micro mammiferi) è il fatto che la ricolonizzazione del Nord Italia da parte della Cicogna bianca è partita proprio dalle aree in cui forte è la presenza dell'attività risicola (Vercellese, Novarese e Pavese).

Tra questi due nuclei attualmente non risultano insediamenti, anche se per l'area nord di Torino viene riportata l'osservazione ripetuta di una coppia nel 2010 a cui non ha fatto seguito l'insediamento e la costruzione del nido. Per favorire l'insediamento sono stati collocati all'interno del Parco Regionale della Mandria alcune piattaforme nido. Tutt'ora non risultano occupati, probabilmente a causa dell'elevata copertura boscosa delle aree circostanti e alla limitata estensione di quelle aperte. Poco distante da questo sito, all'interno dell'Oasi del Lago Borgarino a San Gillio, un'altra piattaforma su palo è stata collocata da un'associazione naturalistica (Ass. Naturalistica Le Gru). Anche in questo caso non vi sono stati tentativi di insediamento da parte di coppie, probabilmente a causa della limitata altezza di tale struttura che non risulta superiore a quelle delle alberature circostanti (parametro fondamentale all'insediamento della specie). Altro fattore che determina l'occupazione di un sito è l'immediata vicinanza di aree aperte allagate, con acqua bassa per lunghi periodi (es. risaie). Questo tipo di ambiente è sicuramente poco comune nel torinese.

La presenza di linee elettriche aeree e tralicci, se da un lato offre un valido supporto per la costruzione del nido, costituisce un pericolo per l'alta possibilità di elettrocuzione. La Cicogna bianca viene classificata come specie estremamente vulnerabile al rischio di folgorazione dall' Association Multidisciplinaire des Biologistes de l'Environnement (A.M.B.E., 1990a, 1990b, 1992, 1993a, 1993b). Le linee più pericolose sono quelle a 15.000 Volt a causa della vicinanza dei fili conduttori (non isolati) all'estremità del palo. Una statistica evidenzia che gli incidenti con linee elettriche aeree costituiscono la causa di decesso del $70 \%$ dei soggetti rilasciati o marcati dal Centro Cicogne di Racconigi (43 individui morti tra il 1985 e il 1997; Vaschetti et al., 2003). Circa il $65 \%$ delle cop- pie in provincia di Torino nidifica su tralicci e questo può rappresentare un fattore limitante all'insediamento della specie e un parametro importante anche per la valutazione dell' idoneità di un sito di reintroduzione o di installazione per strutture artificiali di nidificazione.

Piogge intense e prolungate durante l'allevamento dei pulcini possono influire negativamente sul successo riproduttivo, come rilevato per le coppie di Gassino e di Volpiano dove il maltempo ha causato ripetutamente la morte dei giovani. Come riportato da Belardi, Canziani e Dimitolo non è chiaro se le condizioni meteorologiche avverse agiscano direttamente sullo stato di salute dei piccoli in quanto i giovani implumi nelle prime due settimane di vita hanno grosse difficoltà di termoregolazione $o$, meno verosimilmente, la mortalità post natale sia legata prevalentemente al problema del reperimento del cibo da parte degli adulti.

L'occupazione dei nidi in date piuttosto precoci porta ad escludere la provenienza trans-sahariana di gran parte dei soggetti nidificanti in Provincia e fa propendere a favore di soggetti svernanti in situ.

La presenza sul territorio di disponibilità trofiche durante tutto l'anno, rappresentate soprattutto dalle discariche di rifiuti solidi urbani e il susseguirsi di inverni relativamente miti, ha fatto registrare un costante aumento del numero di Cicogne bianche svernanti in Provincia di Torino, particolarmente a partire dal 2006/2007. Una relazione tra la maggiore sopravvivenza degli adulti e l'incremento della popolazione nidificante è stata individuata per la vicina Svizzera (Schauba, 2004) dove la Cicogna bianca è stata reintrodotta.

La chiusura della discarica di Basse di Stura nel 2010 ha determinato una riduzione momentanea del numero di individui svernanti, limitatamente all'intervallo di tempo necessario alla localizzazione del nuovo impianto di Pianezza. Cicogne bianche sono parimenti presenti in inverno anche presso la discarica di Pinerolo.

La spiccata filopatricità della specie ha determinato la formazione, in Provincia di Torino, di due nuclei relativamente distinti, che frequentano settori diametralmente opposti rispetto all'area metropolitana. Negli ultimi venti anni (1995-2014), il numero di coppie di Cicogna bianca nel torinese è quadruplicato ma la loro sussistenza nel tempo dipende dal mantenimento di condizioni ambientali e trofiche adatte alla specie. La chiusura imminente della discarica di Pianezza, data la messa a regime del nuovo "termovalorizzatore" nel capoluogo, potrebbe influire in maniera negativa sul mantenimento della popolazione in quanto gran parte degli individui nidificanti probabilmente trascorre l'inverno nell'area, alimentandosi in discarica.

L'evoluzione della popolazione torinese dipenderà pertanto da inevitabili interventi di supporto all'insediamento di nuove coppie tramite l'istallazione di piattaforme nido, come già riportato per altri Paesi (Barbraud, 1999; Massemine-Challet et al., 2006), congiuntamente a misure di miglioramento e mantenimento del paesaggio agricolo in aree specificatamente selezionate. E infatti indubbio che la presenza sul territorio, di lunghe linee di elettrodotti, anche in aree favorevoli, possa contrastare negativamente gli sforzi di tutela e conservazione di una specie così sensibile all'elettrocuzione. 


\section{BIBLIOGRAFIA}

Aimassi G. \& Reteuna D., 2007 - Uccelli nidificanti in Piemonte e Valle d'Aosta, aggiornamento della distribuzione di 120 specie. Memorie dell'Associazione Naturalistica Piemontese, 7.

Alessandria G., Della Toffola M. \& Pulcher C., 1994 Resoconto ornitologico del Piemonte e Valle d'Aosta. Anno 1993. Rivista Piemontese di Storia Naturale, 15, 1994: 197-217.

Alessandria G., Della Toffola M. \& Fasano S. G., 2005 Resoconto ornitologico del Piemonte e Valle d'Aosta, Anno 2003. Rivista Piemontese di Storia Naturale, 26, 2005: 321-360.

Alessandria G. et al. 2006 - Resoconto ornitologico del Piemonte e Valle d'Aosta, Anno 2004. Rivista Piemontese di Storia Naturale, 27, 2006: 349-392.

A.M.B.E., 1990a - Lignes moyenne-tension et avifaune. Evaluation des risques d'accidents liés aux pylones et sensibilité des espèces. E.D.F. - Direction de la Distribution.

A.M.B.E., 1990b - Impact potentiel des lignes moyennetension de l'agence d'exploitation Crau-Camargue sur l'avifaune. Proposition de balisage des lignes et equipement des pylones a risques. E.D.F/G.D.F.

A.M.B.E., 1992 - Ligne T.H.T. 225 kV Vielmoulin-Liernais. Impacts prévisibles sur l'avifaune et mesures de réduction d'impacts. E.D.F. - C.E.R.T.

A.M.B.E., 1993a - Reconstruction de la ligne $63 / 90 \mathrm{kV}$ Champvans-Pouilly-sur-Saone, du poste de Pouillysur-Saone au pylone 119. Départements de la Cote d'Or et du Jura. Etude d'impact sur le milieu naturel. Phase A-impact prévisible sur l'avifaune, mesures de reduction d'impact et mesures compensatoires. E.D.F. C.E.R.T.

A.M.B.E., 1993 b - Ligne à 2 circuits 400 kV Grande-IlePiossasco. Impact prévisible sur l'avifaune, mesures de reduction d'impact et measures compensatoires. E.D.F. - C.E.R.T.

Barbraud C., Barbraud J.C. \& Barbraud M., 1999 - Population dynamics of the White Stork Ciconia ciconia in western France. Ibis, 141: 469-479.

Belardi M., Canziani M. \& Dimitolo G., 2004 - La cicogna bianca, storia di un ritorno. Parco Adda Sud.

BirdLife International. 2004 - Birds in the European Union: a status assessment. BirdLife International, Wageningen.

Boano G., 1981 - La Cicogna bianca in Piemonte. Presenza, nidificazione e problemi di conservazione. Rivista Piemontese di Storia Naturale, 2: 59-70.

Boano G., 1992 - Cicogna bianca. In: Fauna d'Italia, 29, Aves. 1. Brichetti P., De Franceschi P. \& Baccetti N. (eds.). Calderini, Bologna: 216-225.

Brichetti P. \& Fracasso G., 2003 - Cicogna bianca. In: Ornitologia Italiana, Vol. 1. Alberto Perdisa Editore, Ozzano dell'Emilia: 124-127.

Caula B. \& Beraudo P.L., 2014 - Ornitologia cuneese. Indagine bibliografica e dati inediti. Primalpe, $\mathrm{Cu}$ neo.

Gustin M. \& Tallone G., 2005 - Censimento della cicogna bianca Ciconia ciconia nidificante in Italia nel 2004. Avocetta, 29: 114.
LIPU \& WWF, 1999 - Nuova lista rossa degli uccelli nidificanti in Italia. Rivista italiana di Ornitologia, 69: 3-43.

Lui F., 2004 - La nidificazione della Cicogna bianca in Italia: cenni storici e situazione attuale. Picus, 58: 136139.

Marotto P., 2015 - Resoconto Provinciale Ornitologico Torinese, Anno 2014. Annuario Torino Birdwatching.

Massemine-Challet S., Gendner JP., Samtmann S., Pichegru L., Wulguè A. \& Le Maho Y., 2006 - The effect of migration strategy and food availability on White Stork Ciconia ciconia breeding success. Ibis, 148: 503-508.

Schauba M., Pradela R. \& Lebretona J.D., 2004 - Is the reintroduced White stork (Ciconia ciconia) population in Switzerland self-sustainable? Biological Conservation, 119: 1105-1114.

Tallone G. \& Camanni S., 1991 - Progetto di reintroduzione della Cicogna bianca (Ciconia ciconia) in Italia: situazione e prospettive. In: Atti del II Convegno Nazionale dei Biologi della Selvaggina. Spagnesi M. \& Toso S. (eds.). Supplemento alle ricerche di biologia della selvaggina, 19: 735-739.

Tallone G., Camanni S., Carpegna F. \& Gariboldi A., 1993 - La Cicogna bianca (Ciconia ciconia L.): un caso di ricolonizzazione recente in Italia. In: Atti VII Convegno dell'Associazione Alessandro Ghigi per la Biologia e la Conservazione dei Vertebrati. Spagnesi M. e Randi E. (eds.). Supplemento alle ricerche di biologia della selvaggina, 21: 239-248.

Toschi A., 1960 - La nidificazione in Italia della Cicogna bianca, del Mignattaio e del Gabbiano comune. Ricerche di zoologia applicata alla caccia, 32: 1-18.

Vanzi R., Ricci M. \& Bottelli F., 1994 - Nidficazione della Cicogna bianca (Ciconi ciconia) nel Piemonte Nord-Orientale dal 1990 al 1994. Rivista Piemontese di Storia Naturale, 15: 163-171.

Vaschetti G. \& Boano G., 2005 - Cicogna bianca. In: Uccelli d'Italia. Spagnesi M.\& Serra L., (a cura di). Ministero dell'Ambiente. Istituto Nazionale Fauna Selvatica. Quaderni di Conservazione della Natura, 22: 77.

Vaschetti G., Fasano S. \& Vaschetti B., 1997 - La Cicogna bianca Ciconia ciconia nel Piemonte Sud-occidentale: parametri riproduttivi nel 1996. Avocetta, 21: 148.

Vaschetti G., Fasano S. \& Vaschetti B., 1999 - La Cicogna bianca Ciconia ciconia nel Piemonte sud-occidentale: parametri riproduttivi nel 1997 e 1998 . Avocetta, 23: 138 .

Vaschetti G., Fasano S. \& Vaschetti B., 2003 - La Cicogna bianca Ciconia ciconia nel Piemonte sud-occidentale: parametri riproduttivi dal 1999 al 2002. Avocetta, 27: 126.

Verner J., Morrison M. L. \& Ralph C. J., 1986 - Modeling habitat relationships of terrestrial vertebrates. University of Wisconsin Press, Madison. 